\title{
Biochemical mechanisms of vitamin A action
}

\author{
By F. Weber, Department of Vitamin and Nutrition Research, F. Hoffmann- \\ La Roche $\mathfrak{G}^{\circ}$ Co. A.G., Grenzacherstrasse 124, $\mathrm{CH}_{4-4002}$ Basle, Switzerland
}

Vitamin $A$ is known to fulfil a number of physiological functions in various animal and human tissues. It is essential for the stimulation of growth and proper development of skeletal tissues, for normal reproduction, for maintenance of rod vision and, most importantly, for the preservation of the differentiated functions of mucus-secreting epithelial tissues (compare recent reviews by De Luca, I977; Ganguly et al. 1980; Navia \& Harris, 1980). Consequently, dietary lack of vitamin A leads to clinical deficiency symptoms such as growth retardation, bone malformation, degeneration of the reproductive organs, night blindness and severe alterations of epithelial structures including xerophthalmia of the conjunctiva and cornea which may result in keratomalacia and finally blindness. Metaplasia of the epithelium does, however, not only occur in the eyes but also in the epithelia of the respiratory, gastrointestinal and urogenital tracts. Histopathologically, the mucus-secreting lining of the epithelia is replaced by a stratified, squamous and keratinizing epithelium, with the exception of the intestinal mucosa which does not keratinize.

Due to these broad physiological functions, vitamin $A$ is one of the most important nutrients for the maintenance of health and life. Therefore, many biochemists have devoted a great deal of their research work to the elucidation of the mode of vitamin A action at the cellular and molecular level. Whereas the direct participation of vitamin $A$ in the process of visual excitation is definitely established, the general molecular function of vitamin $A$ is still being investigated and at present these investigations are strongly concentrated on the fundamental role of vitamin $A$ in cellular differentiation.

In the visual process, vitamin A plays a very special role. This was mainly shown by the brilliant research work of George Wald and his colleagues (Wald, 1968). Vitamin $A$, in the form of its aldehyde, retinal, is bound as a chromophore to the photosensitive pigments rhodopsin and iodopsin which are located in the outer segments of vertebrate rods and cones respectively. Whereas the cones are receptors of light of high intensity and, in the case of human cones, of colour vision, the rods are especially sensitive to light of low intensity. Vitamin A deficiency causes a decrease in the synthesis of rhodopsin and hence creates problems with dark adaptation. Several reactions including oxidation or reduction and configurational changes of the vitamin A molecule take place during the visual cycle. Vitamin $A$ is stored as a long-chain fatty acid ester in the retina pigment epithelium which, in humans, contains the highest concentration of vitamin A apart from the liver (Bridges, 1982). This store probably protects the retina against 
vitamin A deficiency. After hydrolysis of the ester, vitamin A is enzymatically isomerized to $\mathrm{I} 1$-cis retinol and then oxidized to the corresponding I I-cis aldehyde which combines with the protein opsin to form rhodopsin. Light energy bleaches rhodopsin and induces an isomerization of the I I-cis retinal to the all-trans form by simultaneous liberation of the chromophore from the opsin. This energy exchange produces nervous excitation which is transmitted via the optic nerves to the brain, causing visual sensation. The all-trans retinal can either be isomerized to the I I-cis compound to combine again with opsin to form rhodopsin, or it can be reduced enzymatically to all-trans retinol.

This function of vitamin A as the prosthetic group of visual pigments is clearly different from its more general role in epithelial and other tissues of the body. The action of vitamin $A$ in the eye is a passive or subsidiary one. The most interesting question of course is, what is the role of vitamin $A$ in the body besides its role in the visual cycle? For a long time, during the 1950 s and 1960 s, the search for the biochemical mode of systemic vitamin $A$ action had mainly centered on possible involvements of vitamin $A$ in mucopolysaccharide biosynthesis and carbohydrate metabolism, the latter especially in connection with adrenal glucocorticoid biosynthesis (Johnson \& Wolf, r 960; Wolf \& Johnson, r960). The general hypothesis was that vitamin $A$ serves as a coenzyme for ATP sulphurylase (EC 2.7.7.4) and aryl sulphotransferase ( $E C$ 2.8.2.1). Both these enzymes participate in the sulphation of mucopolysaccharides. It was also assumed that vitamin $A$ may have a coenzymatic function for some enzymes which are active in the biosynthesis of adrenocorticoids or important metabolic intermediates (reviewed by Pitt, 1971). However, the role of vitamin $A$ as a simple cofactor in special enzymes could not be established. Only from the beginning of the 1970 s has evidence begun to be accumulated to show that vitamin $\mathrm{A}$ is involved in the glycosylation of glycoproteins at the molecular level (De Luca, Rosso et al. 1970) and is also acting, in some way, on nuclear RNA synthesis (Johnson et al. 1969).

In the following years, a number of studies on the function of vitamin $A$ in the synthesis of glycoproteins clearly demonstrated that, as a result of vitamin A deficiency, there is a reduced biosynthesis of specific glycoproteins in intestinal mucosa (De Luca, Schumacher et al. 1970), corneal epithelium (Kim \& Wolf, 1974), tracheal respiratory epithelium (Bonnani et al. 1973) and liver (De Luca et al. 1975). This decline in glycoprotein synthesis is histologically seen to be concomitant with a reduction in the number of goblet cells in the intestinal mucosa (Olson et al. 198I) and tracheal epithelium (De Luca et al. 1972). The goblet cells produce the mucins, high-molecular-weight glycoproteins, which probably play a role in epithelial surface function.

With regard to the molecular mode of vitamin A action in glycoprotein synthesis, it has been shown by the research groups of De Luca and Wolf (De Luca, Rosso et al. 1970; De Luca, 1977; Wolf, 1977; Adamo et al. 1979) as well as by other groups (e.g., Peterson et al. 1974) that whole cell systems in vivo and in vitro, including hepatocytes, epidermal and intestinal epithelial cells, and also isolated membranes convert retinol into retinyl phosphate. Furthermore, the 
mannosyl residue can be transferred from the activated sugar nucleotide guanosine diphosphate mannose to retinyl phosphate under the formation of mannosyl retinyl phosphate. This retinol-linked mannose complex was, beyond doubt, found to occur in liver and intestinal mucosa of both normal and vitamin A deficient rats after injection of labelled retinol and mannose (Wolf et al. 1979).

Since a significant decrease of incorporation of labelled mannose into liver glycoproteins was observed in both mildly and severely vitamin A deficient hamsters (De Luca et al. 1975; De Luca, I977), it was postulated that mannosyl retinyl phosphate acts as the direct donor of the mannosyl moiety to the oligosaccharide chain of a glycoprotein.

Interestingly enough, this reaction appears to be comparable with another biological mechanism for transferring mannosyl residues. Mammalian membranes also contain a mannolipid wherein the mannosyl group is linked to dolichyl phosphate which was first described 12 years ago (Behrens \& Leloir, I970), almost at the same time as mannosyl retinyl phosphate (De Luca, Rosso et al. 1970). Dolichols, as demonstrated by Morton and co-workers (see Pennock et al. 1960), are widely distributed in nature and represent long-chain polyprenols of varying lengths consisting of fifteen to twenty isoprene units or seventy-five to one hundred $\mathrm{C}$-atoms per molecule. When comparing the structures of vitamin $\mathrm{A}$ and dolichol, vitamin $A$ is chemically closely related to dolichol because vitamin $A$ is a cyclic form of a polyprenol having four isoprene units or twenty $\mathrm{C}$-atoms.

As to the mechanism of mannosyl transfer, however, important differences exist between dolichyl mannosyl phosphate and mannosyl retinyl phosphate. Whereas the mannosyl moiety from dolichyl mannosyl phosphate is transferred to an oligosaccharide chain which is linked to dolichyl pyrophosphate, it has been claimed that mannosyl retinyl phosphate is involved in a direct transfer of mannose to glycoproteins without the formation of an oligosaccharide chain on retinyl phosphate itself. Furthermore, the mannosyl residue from mannosyl retinyl phosphate seems to be incorporated into an oligosaccharide chain which is $O$ glycosidic linked to a serine or threonine molecule of the glycoprotein. However, the preformed oligosaccharide chain combined with dolichyl pyrophosphate is $N$ glycosidic linked with an asparaginyl residue of the peptide core of glycoproteins (De Luca, 1977). The function of glycosyl dolichyl phosphates in the synthesis and transfer of oligosaccharides will be dealt with below.

The principal questions now to be discussed, are: what is the biological significance of the occurrence of mannosyl retinyl phosphate for the biochemical mechanism of vitamin A function? Is this mannosyl transfer, mediated by vitamin $A$, the molecular mechanism for controlling growth and differentiation of epithelial tissues? The answers to these questions are at present not available and remain open. There are many basic problems to be clarified. For example, it is still unknown which energy-rich phosphorylated intermediate is donating the phosphate group to retinol. Until now a kinase for vitamin A has not been described nor has a protein serving as an acceptor of mannose from mannosyl retinyl phosphate been identified or purified. In a recent paper, Quill \& Wolf 
(1981) even reported a non-enzymatic transfer of mannose from mannosyl retinyl phosphate to endogenous protein acceptors. More information is, therefore, necessary with regard to the specificity of the acceptor proteins or to the factors which determine whether the mannosylation of a specific glycoprotein is or is not dependent on mannosyl retinyl phosphate. In other words, is the transfer of mannosyl residues to glycoproteins only a side effect of vitamin A action?

All these questions cannot yet be answered and as long as the metabolic function of vitamin A, expressed in biochemical terms, remains unclear other principles have to be taken into consideration. An interesting hypothesis has recently been proposed (De Luca et al. 1979) concerning a possible role of retinyl phosphate as a carrier of mannosyl residues across the membrane bilayer. In addition it appears quite possible that vitamin A deficiency may provoke a general alteration in the physical characteristics of membranes. Finally, vitamin A may regulate directly the transcription or translation of the protein core of glycoproteins or may influence, by nuclear mediation, the levels of other proteins such as glycosyl transferases or other essential proteins of membranes.

Indeed, more and more evidence indicating a pronounced effect of vitamin $A$ deficiency on RNA synthesis in tissues, such as intestinal mucosa, tracheal epithelium and testes (reviewed by Ganguly et al. 1980) has accumulated in recent years. Chytil and co-workers were able to demonstrate the presence of specific binding sites for retinol in cell nuclei and found in hepatic nuclei from vitamin $A$ deficient animals a strikingly increased specific binding of retinol in comparison with controls (Chytil \& Ong, 1979). These nuclear binding sites for vitamin A appear to be located on the chromatin and it is assumed that the cellular retinolbinding protein delivers specifically the vitamin to the interior of the nucleus whereas the cellular retinol-binding protein itself does not bind to the nucleus or the chromatin (Chytil et al. 1982). In a similar manner to steroid hormones, the interaction of vitamin $A$ with the specific nuclear binding sites probably modulates gene expression as reflected by the observation of characteristic differences between poly-adenosine RNA preparations from testes of control and vitamin A deficient animals.

Recently, another very interesting paper was published by Fuchs \& Green (198I) who investigated the regulation of terminal differentiation of cultured human keratinocytes by vitamin $A$. These authors found that during the course of terminal differentiation in human epidermis, the cells begin to synthesize keratins of a molecular weight greater than 60000 when the cells are moving outwards. On the other hand, the cells located in the deepest layer produce smaller keratin molecules of a molecular weight between 46000 and 58000 . Very significant is the observation that, when culturing the epidermal keratinocytes in the absence of vitamin A, a keratin of 67000 molecular weight was produced. But when the keratinocytes were grown in a vitamin A-containing medium, a smaller keratin of a molecular weight of 52000 was synthesized. In addition, the authors demonstrated that the different keratins were translated from different messenger RNA molecules. Accordingly, this observation documents an excellent example of the 
capability of vitamin $A$ to regulate the synthesis of specific proteins by gene expression, obviously by suppressing the production of large keratin molecules and by promoting the synthesis of a keratin with a smaller molecular weight.

Since the discovery of vitamin $A$ acid, an oxidation product of vitamin $A$, and the demonstration of its vitamin activity (Arens \& van Dorp, 1946), research on the metabolism of vitamin A was markedly stimulated and the search for biologically-active metabolites started. It soon turned out, however, that the efficacy of vitamin A could only partially be substituted by vitamin A acid which was found to be only active in growth promotion and in the differentiation and maintenance of epithelial and skeletal tissues. Vitamin A acid cannot substitute for retinol in maintaining the function of reproductive organs for which the retinol molecule in an unaltered form is obviously necessary. Furthermore, retinoic acid is inactive in the visual process.

There are several key questions concerning the metabolic role of vitamin A acid which still remain to be resolved; for instance, is retinoic acid an obligatory metabolite of vitamin A for its function in certain tissues? Is vitamin A acid effective per se or is it further metabolized to an as yet unknown active form? With regard to the activity of vitamin A to incorporate mannose into glycoproteins, vitamin $A$ acid was shown to be almost as active as retinol (De Luca, I977). However, no evidence could be obtained for the occurrence of mannosyl retinoyl phosphate, being analogous to mannosyl retinyl phosphate, in cultured fibroblasts (Bhat \& De Luca, 1981). Furthermore, it could not be proved in experiments with liver microsomal preparations that chemically synthesized retinoyl phosphate is able to accept mannose and to form the corresponding mannosyl lipid (Frot-Coutaz et al. $198 \mathrm{I}$ ). From these observations the conclusion was drawn that retinoic acid is further metabolized to an as yet unidentified derivative being similar to retinol and containing a hydroxyl group located at the side chain. This hydroxyl group probably enables the metabolite to become phosphorylated and mannosylated as in the case of retinol. It cannot be decided at present whether both retinol and the retinoic acid derivative represent equally active forms of vitamin $A$ in glycosylation reactions. On the other hand, it could also be possible that the action of vitamin $A$ in supporting growth and epithelial differentiation is mainly, if not solely, mediated through the unknown metabolite of retinoic acid (DeLuca, 1979). Lntil now, little has been known about a possible nuclear function of vitamin $A$ acid similar to the alteration of genomic expression by retinol. There are, however, good indications of the existence of nuclear-binding sites for vitamin A acid (Chader et al. 1981).

Investigations in our laboratories are concerned with the influence of vitamin $A$ deficiency on serum lipids, lipoproteins and liver lipids in rats. Approximately 20 years ago, we observed a striking alteration of the rate of incorporation of labelled mevalonic acid into liver cholesterol, squalene and ubiquinones (Wiss et al. 196r). With progressing vitamin A deficiency the mevalonate incorporation into cholesterol dropped markedly whereas more mevalonic acid was incorporated into squalene and ubiquinones. After single, oral administration of vitamin $A$ to the deficient animals, the incorporation rate was reversed: more mevalonic acid was 
incorporated into cholesterol and less mevalonate into squalene and ubiquinones. The same results could be obtained from control and vitamin A deficient rats when labelled mevalonic acid was added to liver homogenates (Weber et al. 1960). Whereas in liver homogenates from controls the radioactive label was equally distributed between cholesterol and squalene, in liver homogenates from vitamin A deficient animals the proportion of radioactivity was higher in squalene than in cholesterol. At that time we concluded that vitamin A interacts in one of the biosynthetic steps for cholesterol following squalene formation. Feedback reactions would result in an accumulation of squalene and also of intermediates between mevalonic acid and squalene yielding, finally, an increased incorporation of mevalonate into ubiquinones, branched-chain fatty acids and polyprenols.

Recently, we started studies in order to elucidate the possible mechanism of the involvement of vitamin $A$ in lipid metabolism. For all experiments, three groups of animals were used (Fig. I), the vitamin A deficient rats receiving a minimum amount of vitamin $A$ just sufficient to prevent the animals from loss of weight and from death, which occurs in completely vitamin A deficient animals within a few days after their growth has ceased. This sudden death of vitamin A deficient animals is mainly due to secondary effects of deficiency. Our aim with this animal model is, however, to deal only with primary effects of vitamin A depletion, these being reversible after supplementary administration of the vitamin.

An analysis of the serum lipids of vitamin A deficient animals in comparison with ad lib.-fed and pair-fed controls revealed that in both control groups all indices measured did not, in principle, differ from each other, thus indicating that the restricted food intake of the pair-fed controls did not influence the biochemical indices. In contrast, body-weight and serum vitamin A levels were, of course,

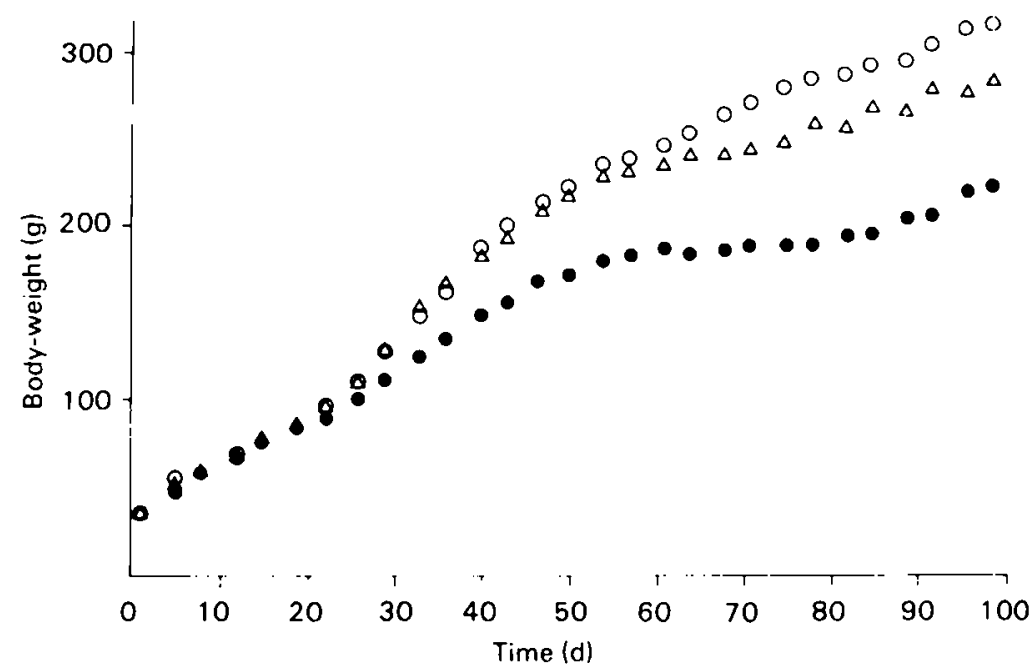

Fig. I. Growth curves of vitamin $A$ deficient and control rats $(O$ ad lib.-fed controls, $\triangle$ pair-fed controls, vitamin A deficient rats). Animals of all groups were kept on a vitamin A depleted diet. On and after the 20 th day each control rat received 140 i.u. of vitamin $A / d$ (orally administered). Each vitamin $A$ deficient animal received a maintenance dose of 2.5 i.u. of vitamin $A / d$. 
decreased in the deficient animals. Simultaneously, $\alpha$-tocopherol levels and, especially, triglyceride levels decreased to about $20 \%$ of normal, whereas phospholipids and cholesterol concentrations were lowered to a lesser extent. The protein levels were not altered by vitamin A deficiency.

The strongly diminished triglyceride levels in the serum of vitamin A deficient rats could be attributed mainly to a tenfold reduction of triglyceride-rich lipoproteins (Fig. 2), the chylomicrons (class o) and the very-low-density lipoproteins (class I). The density class 2 which is rich in cholesterol and includes both the remnants of triglyceride-rich particles and one class of low-density lipoproteins, was practically completely lacking in serum from vitamin A deficient animals. The high-density lipoproteins of density class 4 , mainly containing phospholipids and cholesterol, dropped by about $40 \%$ in comparison with the amount in control animals.

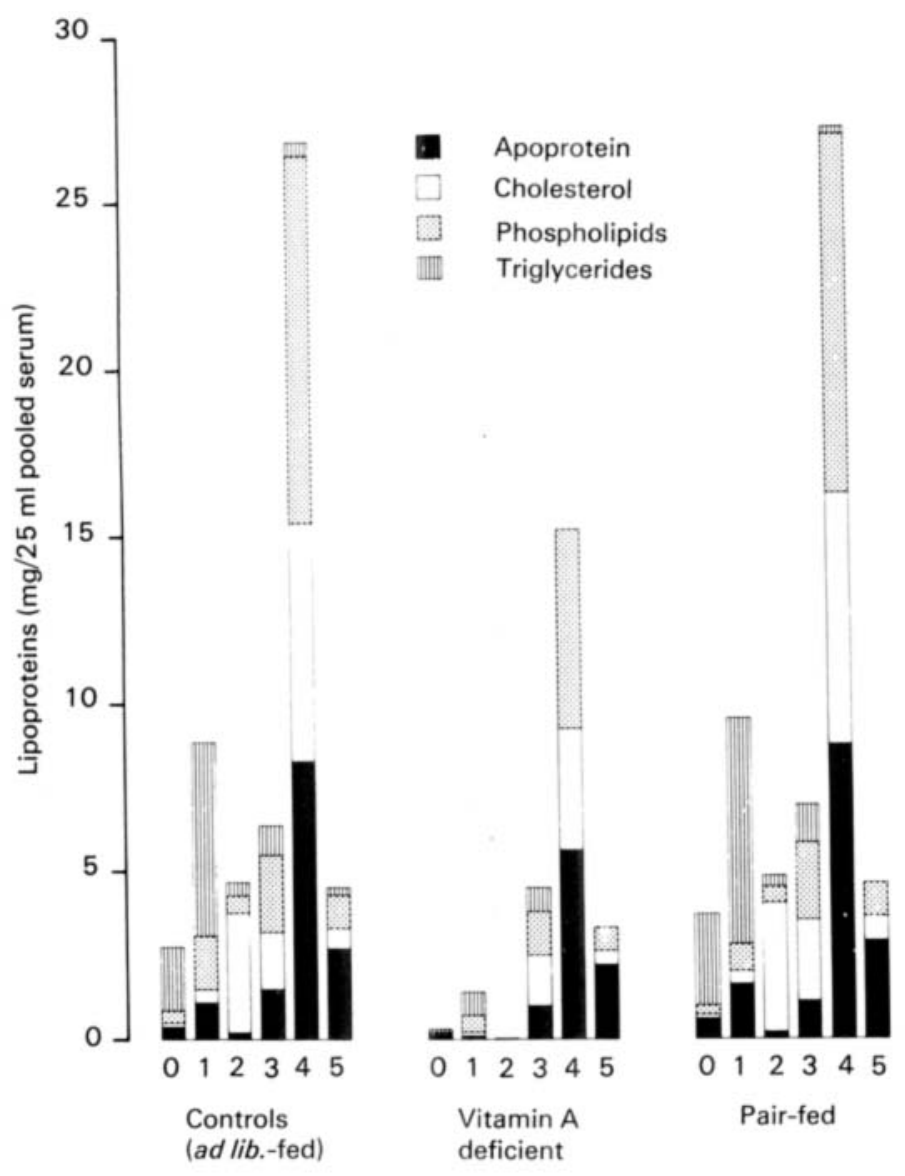

Fig. 2. Distribution of serum lipoproteins in various density classes in control rats (ad lib.-fed and pair-fed) and vitamin $A$ deficient animals. Density classes $0,1,2,3,4$ and 5 represent lipoprotein densities of $<0.95,0.95-1 \cdot 006, \mathbf{1} \cdot 006-1 \cdot 035,1 \cdot 035^{-1} \cdot 05^{6}, 1 \cdot 07^{8-1} \cdot 15^{\circ}$ and I. I 50-I 2 I respectively. 
Results of a lipid analysis in liver of vitamin A deficient, ad lib.-fed and pair-fed animals demonstrated that the hepatic vitamin A store was, of course, completely exhausted in the deficient animals. In contrast to the findings in serum, the $a$ tocopherol, cholesterol and phospholipid levels were not depressed in liver. However, liver triglycerides were found to be reduced too, although not as severely as in serum, whereas the concentration of the dolichols was doubled.

In addition, several hepatic enzyme activities were measured. Phosphofructokinase $(E C$ 2.7.1.56) is considered to play a major role in the regulation of the rate of glycolysis. Its activity is decreased in starvation. Vitamin A deficiency, however, did not alter the enzyme activity, indicating that under our experimental conditions deficiency of this vitamin did not induce starvation. Also citrate synthase ( $E C$ 4.I.3.7), an enzyme of the citric acid cycle, did not respond to vitamin A deprivation. In contrast, the activity of ATP citrate lyase (EC 4.I.3.8) was decreased by about $60 \%$. ATP citrate lyase is a key enzyme in lipogenesis and closely parallels the activity of the fatty acid synthesizing system.

How can these findings be interpreted in terms of a biochemical mode of vitamin $A$ action? Since our studies are continuing, only a preliminary interpretation is possible at present.

One possibility for an interrelationship between vitamin A deficiency and the observed alterations of lipid indices may be associated with the vitamin's function in glycosylation of glycoproteins. An analysis of apoproteins of the phospholipidrich lipoproteins of density class 4 by sodium dodecyl sulphate gel electrophoresis revealed that among the low-molecular-weight $\mathrm{C}$ apoproteins the apo $\mathrm{C}$ III-o band is missing in the sample from vitamin A deficient rat serum. In addition, several bands of apo $E$ polypeptides could not be detected in serum from vitamin $A$ deficient animals. Both apoproteins $\mathrm{C}$ and $\mathrm{E}$ are glycoproteins and are synthesized in the liver. Whereas apo $\mathrm{C}$ contains a relatively large glycosyl portion, the apo $\mathrm{E}$ is glycosylated to a much lesser extent.

When apo $C$ preparations were compared, having similar compositions with respect to the different apo $\mathrm{C}$ polypeptides, their amino acid composition was found to be alike and no difference between vitamin A deficient and control animals was obvious. A marked dissimilarity, however, was observed in the content of some hexosamines in the apoprotein $\mathrm{C}$. The apo $\mathrm{C}$ from deficient animals contained three times as much glucosamine residues as the apo $\mathrm{C}$ from ad lib.-fed or pair-fed rats respectively. The distribution of galactosamine was similar in all three animal groups. On the basis of the results from these experiments we cannot decide whether, in the case of vitamin A deficiency, relatively more glucosamine has been incorporated into the glycosyl chain of apo C than in the controls, or whether the ratio between glucosamine and other hexosamines has been changed in the sense that, except for galactosamine, other hexosamines were incorporated to a lesser degree. Nevertheless, the results indicate an alteration in the glycosylation of apo $\mathrm{C}$.

Similar observations were described by Wolf and his colleagues (Wolf et al. 1979; Kiorpes et al. 1981) who found an under-glycosylation of serum $\alpha_{1}$ - 
macroglobulin in vitamin A deficient rats. Very recently, Rosso et al. (198I) demonstrated that vitamin $A$ deficiency in rats leads to an increased pool of smaller oligosaccharide chains linked to dolichyl pyrophosphate. Whereas in normal liver the glycosyl dolichyl pyrophosphate consisted of three glucose, nine mannose and two $\mathrm{N}$-acetylglucosamine molecules, only five mannoses and two $N$ acetylglucosamines, were bound to dolichyl pyrophosphate in the case of vitamin A deficiency. Normally, the synthesis of the oligosaccharide chain starts from $N$ acetylglucosamine linked to dolichyl pyrophosphate to which another acetylglucosamine and three mannose units are added. The chain is then elongated by further addition of six mannose and three glucose molecules which are provided either by dolichyl mannosyl phosphate or dolichyl glucosyl phosphate, or by other, not yet identified, sugar derivatives. The whole oligosaccharide chain is then transferred to the protein molecule to form a glycoprotein (Hughes \& Butters, I981).

The following results refer again to our own experiments. As in the case of the glycoproteins apo $\mathrm{C}$ and $\mathrm{E}$ in high-density lipoproteins, we could also detect alterations of apoproteins in the very-low-density lipoproteins, namely a change in the ratio of apo $\mathrm{C}$ to apo $\mathrm{E}$ which became smaller in vitamin $\mathrm{A}$ deficient animals compared to normal rats. In other words, when in control animals the ratio between each apo $C$ and apo $E$ polypeptide was approximately $I: 2$, a ratio of $0.5: 2$ was found in vitamin A deficient animals. Based on these results, the following working hypothesis on the effects of vitamin A deficiency on lipid metabolism is proposed.

The very-low-density lipoproteins synthesized in the liver and excreted into the blood stream, as well as the chylomicrons from the small intestine, are known to transfer their triglycerides to endothelial tissues whereby the triglycerides are hydrolysed into fatty acids and glycerol by the activity of lipoprotein lipase. Simultaneously, apoprotein $\mathrm{C}$ and other surface material are delivered from the triglyceride-rich lipoproteins and taken up by high-density lipoproteins. As a consequence of both these reactions, the triglyceride-rich lipoproteins are converted into remnants. These remnants are removed from circulation by their uptake into liver cells which is mediated by a receptor mainly recognizing the apo $\mathrm{E}$ of the remnants. The ratio between apo $\mathrm{C}$ and apo $\mathrm{E}$ is small in remnants. With regard to vitamin A deficiency, our experiments revealed no difference in the net synthesis of nascent triglyceride-rich lipoproteins between deficient and control animals. But we assume that, in contrast to results in control rats, in vitamin A deficient animals the very-low-density lipoproteins are only partly delivering their triglycerides to endothelial tissues, due to a decreased apo $\mathrm{C}$ :apo $\mathrm{E}$ value. As a consequence of this low ratio the very-low-density lipoproteins may behave like remnants and, therefore, may 'erroneously' be taken up again by the liver cells. Accordingly, the triglycerides which were synthesized in the liver return to the hepatocytes and are hydrolysed again to free fatty acids and glycerol. The free fatty acids may inhibit the activity of the ATP citrate lyase by feedback reactions.

Feedback reactions may also be responsible for the alterations of mevalonate 
metabolism as observed in vitamin A deficiency. Lakshmanan et al. (I98I) have recently shown that remnants inhibit cholesterol biosynthesis in the liver at the step of 3-hydroxy-3-methylglutaryl-CoA reductase ( $E C$ I.I.I.34). According to other investigations (Brown \& Goldstein, 1980) a second step of regulating the branched pathway of mevalonate metabolism exists, close to squalene and mediated by cholesterol which is derived exogenously from plasma lipoproteins. Our earlier findings on incorporation of mevalonate into cholesterol and squalene, and also our present observations, are in good agreement with the hypothesis of a multivalent feedback regulation of mevalonate metabolism. We, therefore, assume that the cholesterol synthesis is depressed as a consequence of an increased and artificial reflux of 'wrong' very-low-density lipoproteins to the liver of vitamin A deficient animals. This results in an accumulation of ubiquinones and, as shown in our recent studies, to an increase in the dolichol content in the liver. This finding is especially interesting with regard to the direct participation of the dolichols in the transfer of oligosaccharides to proteins. At present, however, we cannot explain how the accumulation of dolichol in vitamin $A$ deficient livers affects the synthesis of glycoproteins.

In conclusion, we can certainly say that vitamin $\mathrm{A}$, besides its function in rod vision, is involved in the biosynthesis of glycoproteins which are common constituents of membrane systems and which play a key role in many biological processes such as reception of specific hormones, intercellular communication and adhesion, cellular growth, and lipoprotein metabolism. At present, sufficient evidence is not available to decide whether vitamin $A$ is directly participating in the transfer of mannosyl residues, or whether it is otherwise regulating the synthesis of glycoproteins. This regulation might, for example, be mediated by a nuclear function of vitamin $A$ with regard to steering the synthesis of the protein moiety of glycoproteins, or by affecting the synthesis of enzymes involved in the formation of sugar-lipid intermediates such as dolichyl glycosyl pyrophosphate. In addition, it cannot be excluded that both functions, one at the nuclear level by gene expression, and an extra-nuclear effect on glycosylating reactions, are necessary for the overall function of vitamin $A$ in glycoprotein synthesis. It appears, moreover, quite possible that vitamin $A$ exhibits an additional role in certain metabolic events such as release of lysosomal enzymes or regulation of steroidogenesis. In principle, vitamin $A$ most probably fulfils multiple functions at the molecular level which may even be organ-specific. Therefore, it might be difficult to find one common mechanism for the biochemical mode of vitamin $A$ action which could explain, in biochemical terms, all the effects seen in nutritional vitamin $A$ deficiency. Further biochemical and morphological research work on vitamin $A$ is necessary to elucidate its molecular function.

\section{REFERENCES}

Adamo, S., De Luca, L. M., Silverman-Jones, C. S. \& Yuspa, S. H. (1979). F. biol. Chem. 254, 3279 .

Arens, J. F. \& van Dorp, D. A. (1946). Nature, Lond. 158, 622. 
Behrens, N. H. \& Leloir, L. F. (1970). Proc. Natl. Acad. Sci. USA 66, 153.

Bhat, P. V. \& De Luca, L. M. (1981). Ann. N.Y. Acad. Sci. 359, I 35.

Bonnani, F., Levinson, S. S., Wolf, G. \& De Luca, L. M. (1973). Biochim. Biophys. Acta 297, 44 I.

Bridges, C. D. B. (1982). Fedn Proc. Fedn Am. Socs exp. Biol. 41, 860.

Brown, M. S. \& Goldstein, J. L. (1980). F. Lipid Res. $21,505$.

Chader, G. J., Wiggert, B., Russell, P. \& Tanaka, M. (198I). Ann. N.Y. Acad. Sci. 359, I 15.

Chytil, F., Omori, M., Liau, G. \& Ong, D. E. (1982). Fedn Proc. Fedn Am. Socs exp. Biol. 4x, $86 \mathrm{r}$.

Chytil, F. \& Ong, D. E. (1979). Fedn Proc. Fedn Am. Socs exp. Biol. 38, 25 Io.

DeLuca, H. F. (1979). Fedn Proc. Fedn Am. Socs exp. Biol. 38, 2519.

De Luca, L. M. (1977). Vitam. Horm. 35, I.

De Luca, L. M., Bhat, P. V., Sasak, W. \& Adamo, S. (1979). Fedn Proc. Fedn Am. Socs exp. Biol. $3^{8,2535 .}$

De Luca, L. M., Maestri, N., Bonnani, F. \& Nelson, D. (1972). Cancer 30, 1326.

De Luca, L. M., Rosso, G. \& Wolf, G. (1970). Biochem. Biophys. Res. Commun. 41, 6I 5.

De Luca, L. M., Schumacher, M. \& Wolf, G. (1970). F. biol. Chem. 245, 4551.

De Luca, L. M., Silverman-Jones, C. S. \& Barr, R. M. (1975). Biochim. Biophys. Acta 409, 342.

Frot-Coutaz, J., Létoublon, R. \& Got, R. (1981). Ann. N.Y. Acad. Sci. 359, 298.

Fuchs, E. \& Green, H. (1981). Cell 25, 617.

Ganguly, J., Rao, M. R. S., Murthy, S. K. \& Sarada, K. (1980). Vitam. Horm. 38, I.

Hughes, R. C. \& Butters, T. D. (1981). Trends Biochem. Sci. 6, 228.

Johnson, B. C., Kennedy, M. \& Chiba, N. (1969). Am. f. clin. Nutr. 22, 1048.

Johnson, B. C. \& Wolf, G. (1960). Vitam. Horm. 18, 457 .

Kim, Y. L. \& Wolf, G. (1974). F. Nutr. 104, 710.

Kiorpes, T. C., Anderson, R. S. \& Wolf, G. (1981). F. Nutr. 1 x 1, 2059.

Lakshmanan, M. R., Muesing, R. A. \& LaRosa, J. C. (1981). 7. biol. Chem. 256, 3037.

Navia, J. M. \& Harris, S. S. (1980). Ann. N.Y. Acad. Sci. 355, 45.

Olson, J. A., Rojanapo, W. \& Lamb, A. J. (1981). Ann. N.Y. Acad. Sci. 359, 18 I.

Pennock, J. F., Hemming, F. W. \& Morton, R. A. (1960). Nature, Lond. 186, 470.

Peterson, P. A., Nilsson, S. F., Östberg, L., Rask, L. \& Vahlquist, A. (1974). Vitam. Horm. 32, I 8 I.

Pitt, G. A. J. (1971). In Carotenoids, p. 717 [O. Isler, editor]. Basel: Birkhäuser Verlag.

Quill, H. \& Wolf, G. (1981). Ann. N.Y. Acad. Sci. 359, 331.

Rosso, G. C., Bendrick, C. J. \& Wolf, G. (1981). J. biol. Chem. 256, 8341.

Wald, G. ( 1968$)$. Nature, Lond. $219,800$.

Weber, F., Gloor, U. \& Wiss, O. (1 960). Helv. Physiol. Acta 18, C97.

Wiss, O., Gloor, U. \& Weber, F. (196r). Am. F. clin. Nutr. 9, Suppl., 27.

Wolf, G. (1977). Nutr. Rev. 35, 97 .

Wolf, G. \& Johnson, B. C. (1960). Vitam. Horm. 18, 439.

Wolf, G., Kiorpes, 'T. C., Masushige, S., Schreiber, J. B., Smith, M. J. \& Anderson, R. S. (1979). Fedn Proc. Fedn Am. Socs exp. Biol. 38, $254^{\circ}$. 